\title{
Collimated Fast Electron Emission from Long Wires Irradiated by Intense Femtosecond Laser Pulses
}

\section{$\operatorname{AUTHOR}(\mathrm{S})$ :}

Tokita, Shigeki; Otani, Kazuto; Nishoji, Toshihiko; Inoue, Shunsuke; Hashida, Masaki; Sakabe, Shuji

\section{CITATION:}

Tokita, Shigeki ...[et al]. Collimated Fast Electron Emission from Long Wires Irradiated by Intense Femtosecond Laser Pulses. Physical Review Letters 2011, 106(25): 255001.

ISSUE DATE:

2011-06

URL:

http://hdl.handle.net/2433/145928

RIGHT:

(C) 2011 American Physical Society 


\title{
Collimated Fast Electron Emission from Long Wires Irradiated by Intense Femtosecond Laser Pulses
}

\author{
Shigeki Tokita, ${ }^{*}$ Kazuto Otani, Toshihiko Nishoji, Shunsuke Inoue, Masaki Hashida, and Shuji Sakabe \\ Advanced Research Center for Beam Science, Institute for Chemical Research, Kyoto University, Gokasho, Uji, Kyoto 611-0011, \\ Japan and Department of Physics, Graduate School of Science, Kyoto University, Kitashirakawa, Sakyo, Kyoto 606-7501, Japan
} (Received 28 December 2010; published 22 June 2011)

\begin{abstract}
We have experimentally demonstrated that fast electrons emitted from a metallic wire irradiated by a $5 \times 10^{18} \mathrm{~W} / \mathrm{cm}^{2}$ laser pulse can be collimated along the wire, and that their intensity is significantly enhanced in the axial direction of the wire. As the wire length is increased up to $30 \mathrm{~mm}$ from the laser focal spot, the angular divergence of the emitted electrons with energies of hundreds of keV decreases to $65 \mathrm{mrad}$. Numerical simulations reveal that the electrons are trapped by the transient electric field surrounding the wire and guided along the axial direction.
\end{abstract}

Fast electron sources produced through the interaction of relativistic-intensity laser pulses with solid-density targets have been extensively studied because of their potential applications, such as electron diffraction [1-3], particle acceleration [4,5], and fast ignition fusion [6]. Fast electron emission processes including laser acceleration, propagation in plasma, and emission in a vacuum are highly complex, and strongly affected by the characteristics of the laser pulse and target. Many efforts have been made to improve the intensity, directivity, and monochromaticity of electron beams.

Collimated beam generation has been demonstrated experimentally using various targets. A short thin wire attached to a cone-shape target irradiated with a $0.3 \mathrm{PW}$ laser pulse has been used to guide and to collimate electrons in the $\mathrm{MeV}$ energy range [7]. The electrons generated in the cone are guided by the electric and magnetic fields surrounding the wire, which is in the state of plasma. Moreover, a small metal target with a resistivity boundary has been used to collimate electrons by irradiation with a $10^{20} \mathrm{~W} / \mathrm{cm}^{2}$ laser pulse [8]. Using planar targets, collimated beam generation has also been demonstrated [9-15]. For example, a sub-MeV electron beam emitted along the surface of a metal foil target has been observed for laser intensity of $10^{18} \mathrm{~W} / \mathrm{cm}^{2}$; this is interpreted as due to the static electric and magnetic fields generated on the target surface [9]. Such energetic electron transport along the surface has been also studied by observing ions [16] or $\mathrm{x}$ rays [17] emitted from targets. In studies on relativistic laser-solid interactions, such as the above-mentioned works, particle-in-cell (PIC) plasma simulations for a small space (hundreds of micrometers at most) near the laser irradiation spot have been successfully carried out to explain the experimental results. That is to say, such phenomena have been explained by effects in small-scale models.

Here, we experimentally demonstrate collimated electron-beam generation using long metallic wire targets with lengths of the order of centimeters. The characteristics of the electron emission strongly depend on the length of the wire: collimation becomes stronger with increasing wire length. In numerical simulations of electron trajectories based on a simplified model involving a transient electric field decaying in time around the wire, the experimental results for the angular distribution of electron emission are reproduced well.

A Ti:sapphire chirped-pulse amplification system operating with a central wavelength of $800 \mathrm{~nm}$ and a pulse duration of $150 \mathrm{fs}$ is used as the laser source. Laser pulses with a pulse energy of $140 \mathrm{~mJ}$ are focused with an $f / 3.5$ off-axis parabolic mirror to a spot size of $3 \mu \mathrm{m} \times 4 \mu \mathrm{m}$, resulting in a peak intensity of $5 \times 10^{18} \mathrm{~W} / \mathrm{cm}^{2}$. Amplified spontaneous emission is measured to be less than $10^{-7}$ of the peak intensity of the laser pulse. The laser pulse is $p$ polarized and irradiates a tungsten wire with diameter of $300 \mu \mathrm{m}$ at an incidence angle of $45^{\circ}$. Imaging plates (IPs, Fujifilm FDL-UR-V) are used to detect fast electrons. The IPs have high sensitivity in the energy range from 40 to $1000 \mathrm{keV}$ and are most sensitive at around $200 \mathrm{keV}$ [18]. The whole setup is placed in a vacuum chamber with pressure of $0.1 \mathrm{~Pa}$.

Figure 1(a) shows the experimental setup for measuring the angular distribution of electron emission. Wire length $L$ between the laser focal spot and the end of the wire is varied from 2.5 to $30 \mathrm{~mm}$. Double-layer stacked IPs are placed at a distance of $z=150 \mathrm{~mm}$ from the laser focal spot and are covered with aluminum foil of $11 \mu \mathrm{m}$ in thickness in order to prevent exposure of the IPs to light. Figure 1(b) shows typical single-shot images detected with the IPs for $L=2.5,5,10,20$, and $30 \mathrm{~mm}$. On the secondlayer IP, electrons with energies greater than about $400 \mathrm{keV}$ can be detected. The electrons form distinctive ring-shaped patterns on the IPs. The patterns for each wire length are highly reproducible; however, the center of the ring-shaped pattern shows slight shot-to-shot fluctuation within $\pm 30 \mathrm{mrad}$ because its position is sensitive to the 
(a)
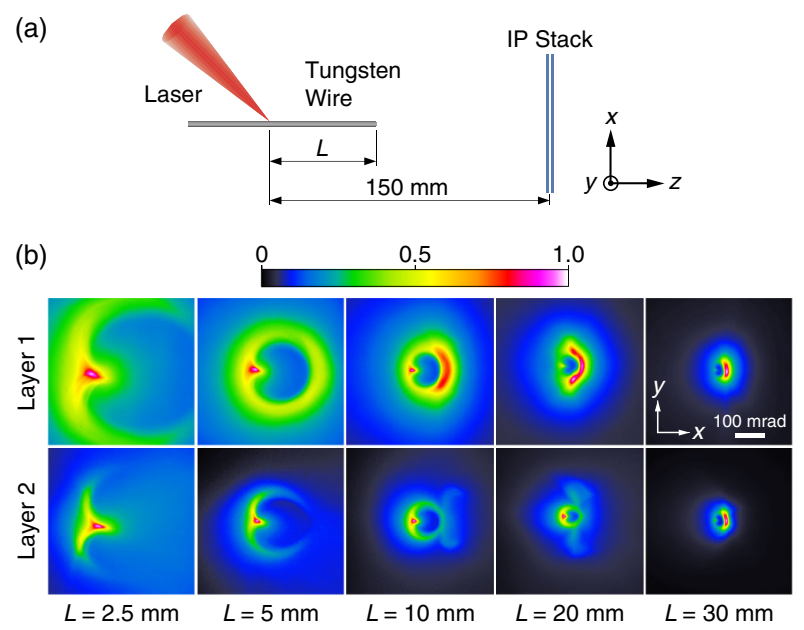

FIG. 1 (color online). (a) Experimental setup for measurement of electron angular distribution using stacked IPs. (b) Single-shot images detected by IPs for $L=2.5,5,10,20$, and $30 \mathrm{~mm}$. The color scale is set independently for each image for maximum contrast. The actual dimensions of the images are $75 \mathrm{~mm} \times$ $75 \mathrm{~mm}$. The center of each image corresponds to the wire axis with an accuracy of $\pm 30 \mathrm{mrad}$.

axial direction of the wire. The overall size of the pattern is drastically reduced as the wire length $L$ is increased. The size of the hole in the ring decreases from 65 to $6 \mathrm{~mm}$ in height as $L$ is increased from 2.5 to $30 \mathrm{~mm}$. The full angle at half-maximum of the electron emission in the horizontal and vertical direction is as narrow as 20 and $65 \mathrm{mrad}$, respectively, at $L=30 \mathrm{~mm}$. In contrast, the IP signal intensity increases with increasing the wire length: the signal intensity at the brightest spot for $L=30 \mathrm{~mm}$ is sevenfold higher than that for $L=2.5 \mathrm{~mm}$. Assuming a typical electron energy to be $300 \mathrm{keV}$ (refer to Fig. 3, described later), the total number of detected electrons is estimated to be of the order of $3 \times 10^{9}$ at $L=30 \mathrm{~mm}$, resulting in laser-to-electron energy conversion efficiency as high as $0.1 \%$.

To improve the collimation of the electron beam, a circular aperture, with diameter of $0.5 \mathrm{~mm}$ in a steel piece of at least 2-mm thickness, is placed at $z=150 \mathrm{~mm}$, as shown in Fig. 2(a). IP1 is used to confirm the aperture position after every laser shot, and IP2 is used to measure the spatial distribution of the electron beam that passes though the aperture. The distance between the aperture and IP2 is $100 \mathrm{~mm}$. The $x$ and $y$ positions of the aperture are adjusted to obtain the highest signal by repeating the measurements; as a result, the aperture is placed a few millimeters away from the wire axis on the $x$ axis. Typical single-shot images detected by IP1 and IP2 for a condition with $L=30 \mathrm{~mm}$ are shown in Fig. 2(b). Three spots (two bright spots and a dim spot) are observed in the image of IP2; furthermore, the distance between the two bright spots is about $3.5 \mathrm{~mm}$, which is considerably larger than the aperture diameter. This therefore shows that the electron (a)

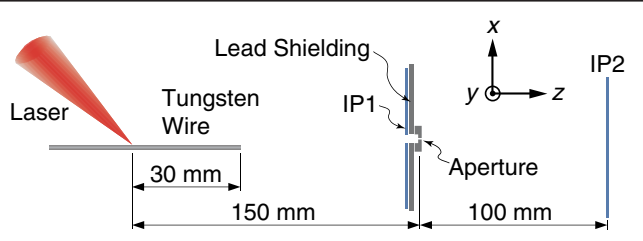

(b)

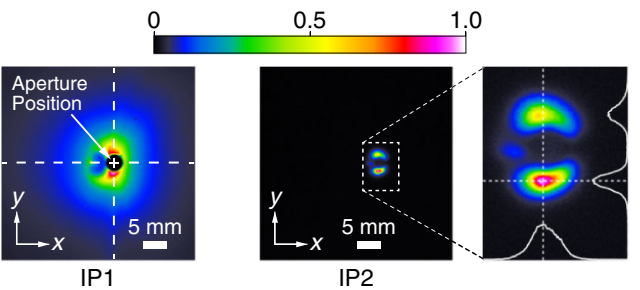

FIG. 2 (color online). (a) Experimental setup for measuring the angular distribution of the electron-beam through a $0.5-\mathrm{mm}$ aperture. (b) The two leftmost images are typical ones obtained by IP1 and IP2. The color scale is set independently in each image for maximum contrast. The actual dimensions of the images are $40 \mathrm{~mm} \times 40 \mathrm{~mm}$. The center of the images roughly corresponds to the wire axis. The rightmost image is an enlargement of a $7.5 \mathrm{~mm} \times 10 \mathrm{~mm}$ area of IP2, along with horizontal and vertical slices of the bright spots.

source cannot be regarded as a small spot, and the electron beam clearly has three directional components. The full width at half-maximum (FWHM) of the lower spot is measured to be $1.6 \mathrm{~mm} \times 0.8 \mathrm{~mm}$, resulting in FWHM of beam divergence of $11 \mathrm{mrad} \times 3 \mathrm{mrad}$. The number of electrons contained in the lower spot is estimated to be $3 \times 10^{6}$ from the energy distribution in Fig. 3 described below.

The energy distribution of the electron beam that passes through the $0.5-\mathrm{mm}$ aperture with the same collimation shown in Fig. 2(a) is measured by using a 0.1-T dipole magnet with $180^{\circ}$ bending angle and an IP positioned in the magnet focal plane. IP1 is also used to confirm the aperture position in the same manner as the previous experiment. Typical distributions of electron energy are shown in Fig. 3(a) for $L=10,20$, and $30 \mathrm{~mm}$. The IP1 images corresponding to each measurement are shown in Fig. 3(b) along with the $x$ and $y$ positions of the aperture. The distributions are broad and have peaks around 200-400 keV. As the wire length is increased, the central energy is slightly shifted to higher energy, and the number of electrons is increased. The number of electrons per unit solid angle subtended by the aperture is estimated to be $7 \times 10^{11} \mathrm{sr}^{-1}$ at $L=30 \mathrm{~mm}$. This value is more than 30-fold higher than the reported one [9], which was obtained by irradiating a planar target at slightly lower laser intensity $\left(2 \times 10^{18} \mathrm{~W} / \mathrm{cm}^{2}\right)$.

Numerical simulations are necessary to understand this phenomenon. However, it should be noted that a rather large area over $30 \mathrm{~mm}$ on the wire target will affect the behavior of the emitted electrons. Two- and threedimensional PIC simulations of laser-plasma interactions have not generally been used for such a large target 
(a)

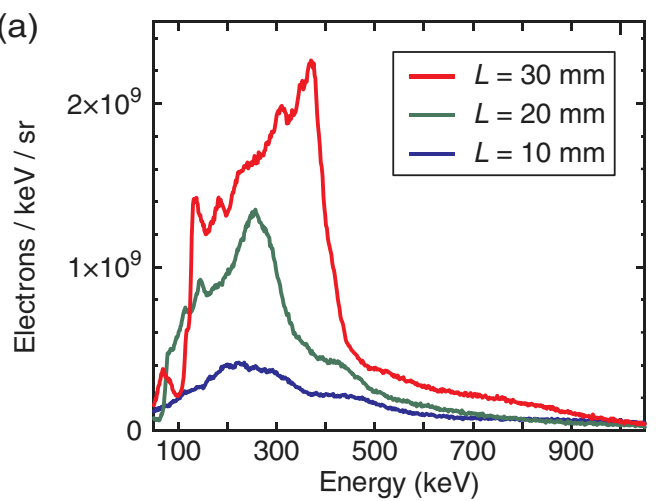

(b)

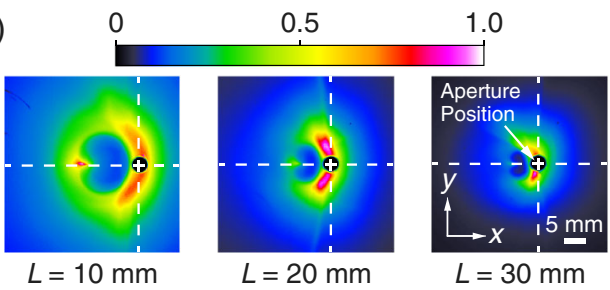

FIG. 3 (color online). (a) Energy distributions of the electronbeam that passes through the $0.5-\mathrm{mm}$ aperture [same collimation as in Fig. 2(a)] for $L=10,20$, and $30 \mathrm{~mm}$. (b) IP1 images corresponding to each energy-distribution measurement. The $x-y$ positions of the aperture are shown for each measurement. The actual dimensions of the images are $40 \mathrm{~mm} \times 40 \mathrm{~mm}$. The center of the images roughly corresponds to the wire axis.

because of computational limits. Here, we perform a threedimensional simulation of electron trajectories in a simplified electric field that approximately simulates the sheath electric field on the target surface. Using proton radiography, Quinn et al. have investigated the transient electric field produced as a result of a laser-wire interaction at $3 \times 10^{19} \mathrm{~W} / \mathrm{cm}^{2}$ [19]. In that work, they observed that an outward-pointing radial electric field as strong as $8 \times 10^{9} \mathrm{~V} / \mathrm{m}$ on the wire surface is produced as a result of the laser-wire interaction and disappears within tens of picoseconds. Referring to those results, we assume that the strength of the radial electric field at a given radial distance $r$ from the axis can be approximated by $1 / r$ outside the wire, and the electric field at a given time $t$ from the laser irradiation can be approximated by $\exp (-t / \tau)$. The simulation is performed using the General Particle Tracer code [20]. All electrons are emitted from an infinitesimal point on the side surface of the wire at $t=0$. The initial energy distribution of the electrons is uniform in the range of $50-1500 \mathrm{keV}$. The angular distribution is uniform on a half-sphere. The electrons injected into the wire area are excluded. The electric field on the wire surface is expressed as a uniform line charge that decays with time. Although the electric field around the wire is in fact not uniform and not electrostatic, this assumption gives a good approximation that the electrons will receive electric forces around a wire from the sheath electric field. When we assume exponential decay $\exp (-t / \tau)$ for the line charge density,

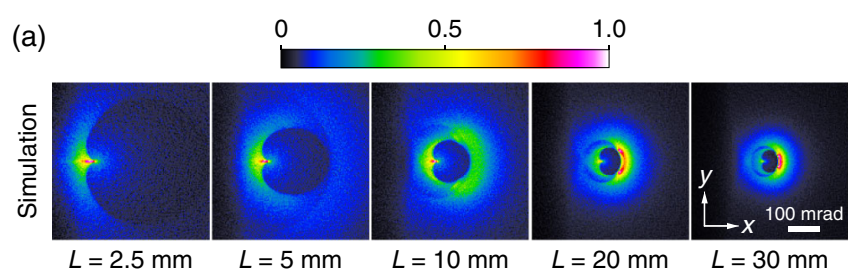

(b)
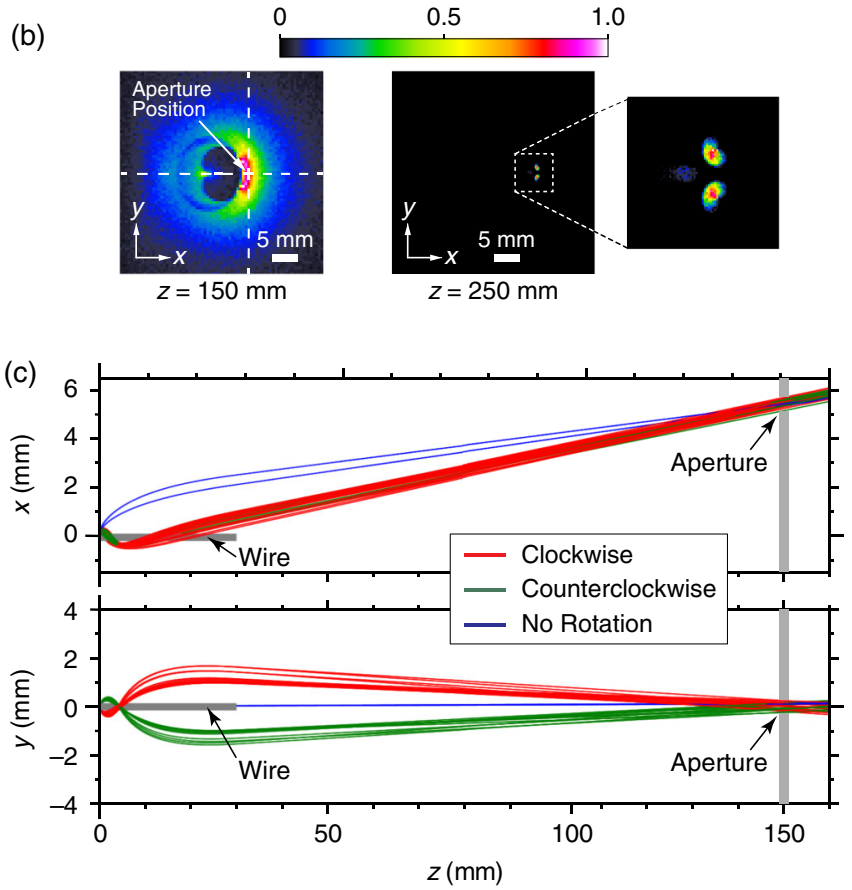

FIG. 4 (color online). Results for numerical simulations of electron trajectories. The $x, y$, and $z$ coordinates correspond to Fig. 1. The center of each image corresponds to the wire axis. (a) Distributions of electrons that hit a screen at a distance of $z=150 \mathrm{~mm}$ from the electron source for $L=2.5,5,10,20$, and $30 \mathrm{~mm}$. Color scale is set independently in each image for maximum contrast. (b) Distributions of electrons that hit a screen at a distance of $z=150$ and $250 \mathrm{~mm}$ from the electron source for $L=30 \mathrm{~mm}$, when an aperture of $0.5 \mathrm{~mm}$ in diameter is placed at $z=150 \mathrm{~mm}$. (c) Trajectories of 30 typical electrons passed through the aperture with the same conditions as Fig. 4(b). The trajectories are classified into three groups: clockwise spiral, counterclockwise spiral, or no rotation.

the distinctive ring-shaped pattern observed in the experiment is roughly reproduced by setting the time constant $\tau$ to be several picoseconds, but the size of the calculated pattern is much larger than the experimental one. In order to reproduce the experimental results well, we introduce exponential decay with two time constants for the line charge density; specifically, the line charge density is given by $\rho(t)=\rho_{1} \exp \left(-t / \tau_{1}\right)+\rho_{2} \exp \left(-t / \tau_{2}\right)$. The unknown parameters are chosen as $\rho_{1}=5 \mu \mathrm{C} / \mathrm{m}, \rho_{2}=1 \mu \mathrm{C} / \mathrm{m}$, $\tau_{1}=5 \mathrm{ps}$, and $\tau_{2}=50 \mathrm{ps}$, resulting in an initial electric field of $7 \times 10^{8} \mathrm{~V} / \mathrm{m}$ at the wire surface.

Figure 4(a) shows the calculated distributions of electrons that hit a screen at a distance of $z=150 \mathrm{~mm}$ from the 
electron source. The distinctive ring-shaped patterns obtained experimentally, shown in Fig. 1(b), are well reproduced for various wire lengths. Moreover, when an aperture with diameter of $0.5 \mathrm{~mm}$ is placed at $z=150 \mathrm{~mm}$ in the same setup as shown in Fig. 2(a), the two bright spots and the dim spot shown in Fig. 2(b) are reproduced well in the simulation [Fig. 4(b)]. The electrons that form the three spots have a broad energy range from 100 to $1200 \mathrm{keV}$, which is roughly consistent with the experimental data. The trajectories of 30 randomly selected electrons that can pass through the aperture are also shown in Fig. 4(c). The calculation results are summarized as follows. (i) The emitted electrons are immediately decelerated by the electric field normal to the wire surface; consequently, the electrons are returned into the wire or guided along the axial direction, and electrons escape from the wire according to their initial energy and direction. (ii) Some portion of the electrons can propagate on spiral trajectories along the wire because of the balance between the centrifugal force and the electrical force. This is the reason that the ringshaped pattern is formed. However, the ring is not axisymmetric, because the electrons are emitted from a point source only on the $x>0$ side, and the number of spiral rotations is one or less for most electrons. (iii) The wire length affects the energy loss that occurs at the wire end. In the case of the short wires, the electrons guided along the wire are decelerated by the axial component of the electric field at the wire end and are spread in the radial directions, because the electrons reach to the wire end before the electric field decreases. (iv) The trajectories can be classified into three groups: clockwise spiral, counterclockwise spiral, and no rotation, as shown in Fig. 4(c). These groups correspond to the three spots observed when the electron beam is spatially selected by the aperture.

In conclusion, we have demonstrated collimated fast electron emission through the interaction of intense femtosecond laser pulses with long metallic wire targets with lengths of the order of centimeters. Emission of electrons with high angular density of nearly $10^{12} \mathrm{sr}^{-1}$ and energies of hundreds of $\mathrm{keV}$ was observed. From the viewpoint of potential applications, since the generated electron beam has a broad velocity distribution and is energy chirped in time, it would be particularly useful as a novel electron source for single-shot time-resolved measurements of ultrafast phenomena, for instance, an electron streak camera
[1] or ultrafast electron diffraction with pulse compression [3]. In regard to another aspect of intense laser interactions, these experimental results proved that large regions of a metallic target can affect electron emission. The electron trajectory simulation, which does not take into account the electron acceleration process in the laser-plasma interaction, reproduced the experimental results well. Therefore, it can be considered that the present phenomenon is strongly related not to the acceleration process, but rather to the target shape.

The authors thank Professor T. Taguchi for valuable suggestions. This work was supported by a Grant-in-Aid for Scientific Research (A) (Grant No. 18206006), a Grantin-Aid for Challenging Exploratory Research (Grant No. 22654050), a Grant-in-Aid for Young Scientists (B) (Grant No. 22760038), a Grant-in-Aid for the Global COE Program "The Next Generation of Physics, Spun from Universality and Emergence" from the Ministry of Education, Culture, Sports, Science and Technology (MEXT), Japan, and Yamada Science Foundation.

*Author to whom correspondence should be addressed. tokita@laser.kuicr.kyoto-u.ac.jp

[1] E. E. Fill et al., Appl. Phys. B 81, 155 (2005).

[2] S. Tokita et al., Appl. Phys. Lett. 95, 111911 (2009).

[3] S. Tokita et al., Phys. Rev. Lett. 105, 215004 (2010).

[4] E. L. Clark et al., Phys. Rev. Lett. 84, 670 (2000).

[5] R. A. Snavely et al., Phys. Rev. Lett. 85, 2945 (2000).

[6] M. Tabak et al., Phys. Plasmas 1, 1626 (1994).

[7] R. Kodama et al., Nature (London) 432, 1005 (2004).

[8] B. Ramakrishna et al., Phys. Rev. Lett. 105, 135001 (2010).

[9] Y. T. Li et al., Phys. Rev. Lett. 96, 165003 (2006).

[10] Z. Li et al., Phys. Plasmas 13, 043104 (2006).

[11] X. H. Yuan et al., Phys. Plasmas 15, 013106 (2008).

[12] L. M. Chen et al., Phys. Rev. Lett. 100, 045004 (2008).

[13] F. Brandl et al., Phys. Rev. Lett. 102, 195001 (2009).

[14] W. Wang et al., Phys. Plasmas 17, 023108 (2010).

[15] G. Y. Hu et al., Phys. Plasmas 17, 033109 (2010).

[16] P. McKenna et al., Phys. Rev. Lett. 98, 145001 (2007).

[17] J. F. Seely et al., Phys. Plasmas 17, 023102 (2010).

[18] A. Taniyama et al., J. Electron Microsc. 46, 303 (1997).

[19] K. Quinn et al., Phys. Rev. Lett. 102, 194801 (2009).

[20] General Particle Tracer code, http://www.pulsar.nl/gpt/. 